\title{
Abducción, método científico e Historia. Un acercamiento al pensamiento de Charles Pierce
}

\author{
Abduction, the scientific method and history. An approach to the thoughts of \\ Charles Peirce
}

\author{
María del Carmen Pía Martín \\ Universidad Nacional de Rosario - \\ Instituto Educación Superior $\mathrm{N}^{\circ} 28$ \\ mpmartin1@hotmail.com
}

\section{Resumen}

El trabajo se propone analizar las formas de inferencia propuestas por Charles Peirce y, en particular, la importancia que adjudica a la abducción, a fin de reflexionar sobre su utilidad para la investigación científica y su aplicabilidad en el campo de la Historia. Según Peirce, la abducción es el proceso por el cual se forma la hipótesis explicativa. Su relevancia procede de la convicción de que es el único modo de inferencia que introduce una nueva idea, siendo fuente de otras hipótesis y, por tanto, tiene un sentido claramente creativo, con todo lo que esto significa para el avance del quehacer científico. En este marco, nos proponemos considerar los aportes posibles al método histórico. El presente artículo, luego de analizar la abducción en términos lógicos y metodológicos, intentará una transposición a las Ciencias Sociales y, en particular, a la Historia.

\section{Palabras clave}

Charles Peirce, abducción, método científico, historia

\begin{abstract}
This work's intention is to analyze the forms of inference proposed by Charles Peirce and the importance it assigns to abduction in particular, in order to reflect on its utility to scientific investigation and its applicability in the field of history. According to Peirce, abduction is the process through which explanatory hypotheses are formed. Its relevance comes from the certainty that it is the only form of inference that introduces a new idea; it is a source of other hypotheses and, therefore, it has a clearly creative direction, with everything that means to the progress of scientific work. In this framework, we propose to consider possible contributions to the historical method. In this article, after analyzing abduction in logical and methodological terms, we will attempt to transpose it to the social sciences and to history in particular.
\end{abstract}

\section{Key words}

Charles Peirce, abduction, scientific method, history

Esta obra está sujeta a la Licencia Reconocimiento-NoComercial-Compartirlgual 4.0 Internacional de Creative Commons. http://creativecommons.org/licenses/by-nc-sa/4.0/ 


\section{Abducción, método científico e Historia}

\section{Introduccción}

El propósito del presente trabajo es realizar un análisis de las formas de inferencia propuestas por Charles Peirce. Centraremos nuestro interés en la abducción, con el objeto de considerar su utilidad para la investigación científica. Según Peirce, la abducción sería el proceso por el cual se forma la hipótesis explicativa ${ }^{1}$. El aporte de este autor consiste en señalar, desde fines del siglo XIX, que la hipótesis es otra forma de inferencia, al igual que la inducción y la deducción, con lo cual modifica y complejiza la noción de método científico. ${ }^{2}$ Por otro lado, la relevancia del tema procede de la convicción -suficientemente destacada por el autor y sus analistas- de que la abducción es el único modo de inferencia que introduce una nueva idea, siendo fuente de otras hipótesis y, por tanto, su función es claramente creativa ${ }^{3}$, con todo lo que esto significa para el avance de la ciencia.

Por nuestra formación profesional, la perspectiva adoptada tenderá a considerar los posibles aportes al método histórico. De tal forma desarrollaremos: a) el concepto de abducción; b) las diferencias y semejanzas con las demás formas de inferencia y con la analogía; c) la consideración de la abducción en términos metodológicos y su transposición a ciertos pasos que resulten adecuados para las Ciencias Sociales y, en particular, para la Historia.

\section{El concepto de abducción}

Peirce descubrió la abducción partiendo del estudio del silogismo y luego fue distanciándose de él. Su hallazgo se llevó a cabo mediante un proceso por el cual, progresivamente, consiguió diferenciar con mayor precisión dicho concepto. Varios autores coinciden en que tal evolución puede dividirse en antes y después

\footnotetext{
1 Charles S. Peirce, "Lecciones de Harvard sobre el pragmatismo, Lección IV: Tres tipos de inferencia", 1903, 4.21. Traducción Castellana y notas: José Vericat, 1988. http://www.unav.es/gep/OnThreeTypesReasoning.html

${ }^{2}$ El debate sobre la importancia de la hipótesis fue introducido por el matemático Henri Poincaré en 1845. Hasta ese momento dominaba la idea de que la función del científico era descubrir las regularidades existentes en la naturaleza de las cosas. El método partía entonces de la observación y, mediante la inferencia inductiva, se pasaba a la generalización, formulando leyes inmutables. La exaltación de este procedimiento también alcanzó a las Ciencias Sociales, en un impulso por proporcionar mayor rango científico a disciplinas siempre amenazadas por la subjetividad. La tesis de Poincaré, en cambio, planteaba que las proposiciones generales de los científicos, o son meras definiciones o convenciones acerca del uso del lenguaje, o son hipótesis encaminadas a cristalizar y organizar un pensamiento ulterior, sujeto a verificación, modificación y refutación. Con esto abría el camino a dos preocupaciones que acompañarían a Peirce en sus estudios: el enfoque semiótico y una lógica del método científico que revisaría el lugar correspondiente a la hipótesis.

${ }^{3}$ Charles S. Peirce, op. cit.; Lúcia Santaella, "La evolución en los tres tipos de argumentos: abducción, inducción y deducción", en CS Peirce y la abducción. Analogía Filosófica XII/1, 1998, 1-187. http://www.unav.es/gep/AN/ANIndice.html
} 


\section{María del Carmen Pía Martín}

de 1900. En la medida de lo posible, tendremos en cuenta este dato a fin de evitar interpretaciones erróneas en nuestro trabajo.

Para Charles Peirce, todo conocimiento procede de la transformación o perfeccionamiento de otro previo. Por tanto, todo conocimiento es inferencial. Los dos modos básicos de inferencia considerados en la tradición científica occidental han sido, por largo tiempo, la deducción y la inducción. Sin embargo, desde 1865, aproximadamente, nuestro autor distinguió la abducción como una tercera forma de inferencia bien diferenciada. En la segunda etapa de su trabajo sobre el tema, pasaría a definirla como el primer paso del método científico. ${ }^{4}$

Ya hemos dicho que su punto de partida fue la lógica clásica. Su estudio del silogismo aristotélico le permitió pensar que, además de la deducción, se podrían formalizar dos modos más de razonamiento, aunque con distintos niveles de veracidad. La deducción es el razonamiento necesario, apodíctico, que se desplaza de lo general a lo particular; es explicativo, pues explicita lo que está contenido en las premisas, proporciona el máximo grado de certeza, pero no agrega nada. Más adelante, formularía la deducción como la inferencia de un resultado a partir de una regla y un caso.

Peirce pensó que, invirtiendo la forma de la deducción, es decir, colocando en la conclusión la premisa mayor de la deducción, se obtiene un silogismo de la inducción. No es un silogismo correcto, porque no se puede concluir una proposición universal a partir de dos particulares. Para que la inducción fuera necesaria, habría que enumerar el total de individuos posibles. Un razonamiento inductivo sólo puede conducir a una convicción razonable, que permite establecer normas o elementos de orientación general. Pero, en términos lógicos, es un modo de argumentar distinto, bien diferenciado. Además, es un pensamiento sintético y ampliativo, es decir, agrega información porque lo que dice en la conclusión no estaba en las premisas. Va de lo particular a lo universal o, en otros términos, es la inferencia de una regla general a partir de un caso y un resultado.

Finalmente, existe una tercera forma de inferencia, que Peirce creyó haber descubierto en lo que Aristóteles denominó “apagoge”. Se corresponde con lo que Peirce llamó, al comienzo, hipótesis, conjetura o suposición; luego preferiría los términos "retroducción" y, sobre todo, “abducción”. En la hipótesis se pone como conclusión lo que en la deducción era la premisa menor. Aunque sean ciertas las premisas, la conclusión es sólo probable. Las premisas sugieren la explicación, pero la respuesta puede ser otra no tenida en cuenta. Por tanto, en este razonamiento no hay necesidad. La abducción sería también un modo sintético o ampliativo en la medida que permite sumar conocimiento. En definitiva, la abducción es la inferencia de un caso a partir de una regla general y un resultado. ${ }^{5}$

En realidad, la hipótesis busca explicar la causa de un hecho sorprendente o, en palabras de Peirce, se detiene en "una circunstancia muy curiosa, que se

\footnotetext{
${ }^{4}$ Gonzalo Génova, "Los tres modos de inferencia”, en Anuario Filosófico XXIX/3, 1996, http://www.unav.es/gep/AF/AFIndice.html; Lúcia Santaella, ed. cit. ${ }^{5}$ Ibidem.
} 
explicaría por la suposición de que fuera un caso de cierta regla general. 0 bien cuando constatamos que en ciertos aspectos dos objetos guardan una marcada semejanza, e inferimos que asemejan entre sí notablemente en otros aspectos" 6 . En esta definición hay planteadas dos posibilidades: la segunda de ellas tiene carácter analógico. Según Guy Debrock, la abducción por analogía no sería una verdadera abducción, sino una especie de deducción disfrazada y la llama "abducción débil". Este autor considera que marcar esta distinción permite formular una idea más precisa del razonamiento abductivo. Así, lo que él denomina "abducción fuerte" se caracteriza por la ausencia de analogía. ${ }^{7}$ Sin embargo, otros autores destacan la importancia de la relación entre abducción y analogía planteados por el propio Peirce. ${ }^{8}$ Más adelante volveremos sobre esta cuestión.

Nos interesa destacar aquí que, luego de 1900, Peirce definió el método científico partiendo de la abducción y en este sentido consideramos que su aporte es sustancial. Se podría decir que Peirce avanzó de una cierta confusión entre inducción y abducción, en tanto pensamientos sintéticos, a una clara distinción y rearticulación de la relación existente entre ambos. Por último, en 1901 definió los sucesivos pasos del método científico considerando la abducción como el momento inicial de adopción de hipótesis, a partir de la cual se utilizan el razonamiento deductivo para la predicción de sus consecuencias posibles y la inducción como fase comprobatoria, ahora nítidamente diferenciada. De este modo, se modificaba la idea de que el proceso inductivo conducía a la formulación de hipótesis partiendo de la observación, tomando distancia del positivismo 9 . Para Peirce, sería el proceso abductivo el que permitiría formular hipótesis e, incluso, elaborar teorías que luego serían desarrolladas y comprobadas por medio de mecanismos deductivos e inductivos. De esta forma, a nuestro juicio, Peirce otorga una centralidad inédita a la abducción en las posibilidades de invención y de progreso del conocimiento científico. A tal punto es la importancia otorgada a la hipótesis, que llegó a presentarla como la clave de su pragmatismo, en tanto es ella la que nos mueve a formar hábitos para actuar en la realidad. ${ }^{10}$

\footnotetext{
${ }^{6}$ Charles S. Peirce (2.624), en Gonzalo Génova, ed. cit.

7 Guy Debrock, "El ingenioso enigma de la abducción", en CS Peirce y la abducción..., ed.cit.

8 Mauricio Beuchot, "Abducción y analogía”, en CS Peirce y la abducción..., ed.cit.

${ }^{9}$ Algunos autores señalan que la inducción requiere, en su inicio, ciertos principios generales - la uniformidad de la naturaleza, las variedades limitadas- para garantizar sus inferencias; ellos también son enunciados universales obtenidos de la observación : “...el principio de la inducción debe ser, a su vez, un enunciado universal, de modo que si procuramos considerar que su verdad ha sido establecida mediante la experiencia, se presentarán de nuevo los mismos problemas, precisamente, que motivaron su introducción" cayendo, por tanto, en el regreso al infinito. (Félix Gustavo Schuster, El método en las Ciencias Sociales, Buenos Aires, CEAL, 1992, p. 56). Nosotros consideramos que, definido así el método inductivo, se distingue de la abducción. Aun cuando la hipótesis requiera de una abstracción o generalización -como sostiene Beuchot-, ésta debe ser entendida como un acto surgido del hecho sorprendente; por tanto, nos parece distinta de la generalización previa aludida más arriba, que pauta las condiciones y la validez del proceso inductivo.

10 Mauricio Beuchot, ed. cit.
} 


\section{María del Carmen Pía Martín}

\section{Abducción, inducción y analogía}

A partir de 1878, Peirce comenzó a despreocuparse de las semejanzas, profundizando su interés por las diferencias entre abducción e inducción. Esta distinción parece importante para clarificar el concepto mismo de abducción.

Según Gonzalo Génova, la inducción y la abducción tienen en común su carácter ampliativo, destacado por Peirce en la etapa inicial de sus estudios. Sin embargo, mientras la inducción va de lo particular a lo general - clasifica -, la abducción es una forma de inferencia que conduce del efecto a la causa - explica -. Mientras la primera es capaz de clasificar hechos no observados, formulando una ley que generalice por la semejanza con los hechos observados, la abducción recurre a un hecho distinto que explica el hecho observado. La hipótesis, a decir de este autor, sería un paso más temerario y peligroso. Por otro lado, la abducción se centra en los caracteres, mientras la inducción lo hace en los individuos. Mientras los individuos pueden enumerarse, los caracteres no. Esto supone una modificación en el razonamiento empleado para la inducción. ${ }^{11}$

Sin embargo, Génova destaca que en ocasiones la inducción también participa de la naturaleza de la hipótesis: un razonamiento puede a la vez ampliar al conjunto de individuos y explicar las causas de un fenómeno a partir de sus efectos. Es decir, reconoce en la formulación de Peirce una especie de mezcla entre inducción y abducción para cierto tipo de casos, sobre todo los que se relacionan con la física. Por el contrario, Lúcia Santaella, considera que esta confusión responde a una instancia evolutiva en pensamiento de Peirce y destaca que después de 1900 la distinción es nítida: la abducción es el razonamiento que lleva a la adopción de una hipótesis y la inducción sirve para su comprobación. Lo que antes llamaba hipótesis, ahora sería considerado como un tipo de inducción, la inducción cualitativa y lo que consideraba inducción, pasó a ser una inducción cuantitativa. Al mismo tiempo, habría acentuado su interés en la función metodológica de los modos de inferencia, por encima de la cuestión comprobatoria. Las inferencias como acciones mentales quedarían así claramente atadas a la noción de método científico y a sus mismos límites. ${ }^{12}$

Por último, para Mauricio Beuchot ${ }^{13}$, mientras en la inducción la cuestión consiste en comprender que todas las As son Bs, la abducción supone un paso previo: captar que las As tienen algo que ver con las Bs. Su enfoque también acentúa la función metodológica de la abducción y trata de rescatar la conexión entre ésta y la analogía. Plantea así que la abducción, para que sea útil, debe llevar implícito un acto de abstracción o generalización, y esto justificaría el considerarla como una forma inferencial cuyas condiciones de realización dependen de la analogía y pueden enseñarse. Coincide con otros autores, entonces, en que la abducción supone una forma lógica, aunque parezca contradecir los principios

\footnotetext{
11 Gonzalo Génova, ed. cit.

12 Lúcia Santaella, ed. cit.

13 Mauricio Beuchot, ed. cit.
} 


\section{Abducción, método científico e Historia}

elementales de la lógica. ${ }^{14}$ Pero para él, esa logicidad nace de un buen manejo de la analogía, de otra forma no sería posible realizar abducciones adecuadas. Es la analogía la que otorga a la hipótesis su carácter abstractivo $\mathrm{y}$, por tanto, de generalidad. El empleo de la abducción, con un fondo analógico, permite universalizar sin olvidar lo particular, y proporciona un margen amplio para explorar el campo del conocimiento, pero a la vez lo hace perfectible y correctible, sobre la base no sólo de las semejanzas, sino también de las diferencias.

En esto contrasta con Debrock ${ }^{15}$ quien, como ya hemos visto, pretende una superación de la relación entre abducción y analogía, para dar más especificidad a la primera. Considera que existen casos tanto de inducción como de abducción débiles que son en realidad formas de deducción encubiertas. Precisamente, a su juicio, Peirce habría evolucionado de las formas débiles, en su etapa ligada al silogismo -viendo inducción y abducción como deformaciones de la deducción- a las formas fuertes. Las abducciones e inducciones propiamente tales las considera "fuertes". Y ambas no pueden confundirse entre sí, son distintas.

Por otro lado, considera más difícil distinguir una abducción fuerte que una inducción fuerte. Para varios autores, la definición más precisa de la abducción, en Peirce, corresponde a su madurez pragmaticista, y la define así:

"Un número de hechos son conocidos. Suponiendo un determinado estado de cosas (que hasta el momento no ha sido probado ni percibido), los hechos conocidos se seguirían de aquél. Luego hay una buena razón para suponer que el susodicho estado de cosas es real. (CP 5.189, 5.197, 1903)"16

Formulado de este modo el problema, se pone el acento en el carácter explicativo de la hipótesis. Según Debrock, esta definición peirceana se coloca fuera de toda base analógica. Por tanto, no encubre un razonamiento cuasideductivo, sino que se emancipa de él. Y ésa es la clave del análisis de este autor: la abducción es distinta de la inducción, y para que sea tal no puede haber ni deducción disfrazada ni base analógica en ella. Por el contrario, debería ser explicada por un "misterioso flash de entendimiento", que analizaremos más adelante.

\section{Las tres formas de inferencias y el método científico}

Hasta el momento hemos visto que la mayoría de los autores coinciden en destacar la abducción como un modo de inferencia que es formulado con más exactitud por un Peirce maduro. Ella gana autonomía y se convierte en un paso del método científico, el inicial, clave para el desarrollo de teorías y fundamental para entender el pragmaticismo peirceano. Este enfoque constituiría una de las

\footnotetext{
${ }^{14}$ Cfr. Guy Debrock, ed. cit.

15 Ibídem.

16 En Guy Debrock, ed. cit.
} 


\section{María del Carmen Pía Martín}

variantes de lo que puede considerarse una lógica o, en sentido más amplio, una metodología del descubrimiento. ${ }^{17}$

En 1901, nuestro autor describió con claridad la relación de la abducción con la deducción y la inducción, presentándola como un método en sus sucesivos pasos: "Cuando surgen hechos sorprendentes se busca una explicación debe ser una proposición tal que lleve a la predicción de los hechos observados, sea como consecuencias necesarias, sea al menos, como muy probables en esas circunstancias. Entonces ha de adoptarse una hipótesis que sea en sí misma plausible y que torne los hechos plausibles. Este paso de adoptar una hipótesis como sugerida por los hechos es lo que llamo abducción". (CP 7.202, c.1901) Y agrega: "En cuanto la hipótesis ha sido adoptada la primera cosa que hay que hacer es delinear sus consecuencias experimentales necesarias y probables. Este paso es una deducción" (CP 7.203, c.1901) "El paso siguiente es la verificación de la hipótesis a través de experimentos comparaciones de las predicciones deducidas de la hipótesis con los resultados reales del experimento. Cuando predicciones tras predicciones son verificadas por el experimento, comenzamos a darnos cuenta de que la hipótesis puede contarse como un resultado científico. 'Este tipo de inferencia, comprobar predicciones basadas en una hipótesis mediante experimentos, es la única que está legitimada para ser llamada propiamente inducción'”. (CP 7.206, c.1901)18

De esta definición se desprende con claridad la reformulación del método, considerando que frente a un hecho sorprendente, partimos de hipotetizar, luego derivamos deductivamente las consecuencias posibles de esa hipótesis y por último comprobamos experimentalmente, recurriendo a la inducción. Según Santaella, en su evolución, Peirce llegaría a formular distintos estadios de investigación donde la abducción representa la primeridad, la deducción la secundidad y la inducción la terceridad, fundado en su orden de interdependencia en el proceso. ${ }^{19}$

La abducción sería así el primer paso pero, a la vez, al ser una forma de inferencia más, debe reconocer una lógica propia, que la haga aprehensible y por tanto resulte de utilidad. No puede fundarse en el azar ni en una total arbitrariedad. El propósito de Peirce al estudiar la abducción fue "dar un instrumento a la lógica de la invención". Se propuso razonar "hacia la hipótesis, esto es, desde los hechos hacia la hipótesis que les señala su causa o los explica”. ${ }^{20}$ Así,

\footnotetext{
${ }^{17}$ Félix Gustavo Schuster, op.cit., pág. 16-17.

${ }^{18}$ Citado en Lúcia Santaella, ed. cit.; Cfr. Charles Peirce, Sobre la lógica de la extracción de la historia a partir de Documentos Antiguos, especialmente testimonios, 1901. Traducción castellana de Douglas Niño, 2001, pág. 24 - 25 y 27.

https://www.google.com.ar/?gws rd=ssl\#q=Sobre+la+L\%C3\%B3gica+de+la+Extracci\%C3\%B3n+ de+la+Historia+a+partir+de

${ }^{19}$ La primeridad la constituyen aquellos fenómenos que se presentan sin tener relación con otra cosa, es pura posibilidad; la segundidad indica polaridad, relación entre dos cosas, realidad; mientras la terceridad se define por la relación de tres elementos, es la categoría del pensamiento, la generalidad o la ley. (Wenceslao Castañares, "El efecto Peirce. Sugestiones para una teoría de la comunicación", en Anuario filosófico XXIX/3, ed.cit.; cfr. Lúcia Santaella, ed. cit.)

${ }^{20}$ Mauricio Beuchot, ed. cit.
} 
creemos necesario considerar qué es un hecho sorprendente, cuáles son los hechos sobre los que se puede hipotetizar, qué condiciones son adecuadas para ello, en definitiva, cómo se produce el proceso abductivo en términos lógicos y cuál es su aplicabilidad a nivel metodológico.

\section{El hecho sorprendente}

Nuevamente, en la consideración de los fenómenos sobre los que nos es posible hipotetizar, Peirce toma distancia del positivismo. Para él, los tipos de hipótesis que pueden formularse para explicar un hecho observado son tres:

a) Las que se refieren a hechos no observados pero susceptibles de verificación en el futuro.

b) Las hipótesis sobre hechos que no pueden ser observados porque pertenecen al pasado. Serían observables en principio, ya que quizás fueron contemplados por alguien, pero no pueden repetirse porque ya se extinguieron. Se ajustan a fenómenos de las Ciencias Naturales, por ejemplo, los estudios de fósiles y geológicos; o la Historia, cuyos hechos pertenecen al pasado y no podrían repetirse en idénticas condiciones. No obstante, hay indicios -documentos, monumentos, tradiciones - que nos inducen a admitir su existencia.

c) Los hechos, fenómenos o entidades que, en el actual estado de desarrollo del conocimiento, no son ni teórica ni factualmente observables.

Es decir, para Peirce, la ciencia no sólo trabaja con hechos que pueden observarse en forma directa, sino que continuamente - y cada vez más - opera sobre realidades que no lo permiten y recurre a hipótesis para explicarlas. Si bien todo científico procura no perder de vista la experiencia sensible, la trasciende para captar su racionalidad última. ${ }^{21}$

Deberíamos considerar ahora cuándo un hecho puede considerarse sorprendente desde una perspectiva peirceana. Un hecho externo resulta sorprendente cuando se presenta como un fenómeno anómalo, que induce a la búsqueda de una explicación, es decir, a la formulación de una hipótesis. Sin embargo, esto merece más precisiones. Según Michael Hoffmann, en la epistemología de Charles Peirce, toda cognición está mediada por signos o elementos de generalidad. A su vez, tal forma de cognición nunca se da en estado puro, sino que está siempre determinada por percepciones, perspectivas, teorías previas. Es decir, cada cognición está inserta en un conjunto de contextos. ${ }^{22}$ Estos contextos - sociales, naturales, de saber hacer práctico - actúan como reglas o leyes que condicionan la posibilidad de percepción y de razonamiento, dentro de

\footnotetext{
${ }^{21}$ Gonzalo Génova, ed. cit.

${ }^{22}$ Para este autor, el contexto es un general que interactúa con el evento concreto, particular. Es una relación de mutua dependencia entre hábitos de entidades diversas. Y proporciona como ejemplo la co-evolución del caballo y la sabana, que se modifican mutuamente. (Michael Hoffmann, "¿Hay una lógica de la abducción?", en CS Peirce y la abducción.., ed.cit.)
} 


\section{María del Carmen Pía Martín}

una evolución temporal, ya que no son eternos ni estáticos. El hecho sorprendente peirceano debe situarse en esta perspectiva: es experimentado como pura segundidad; carece de ciertas expectativas dadas en el marco de determinado contexto de creencias, aunque por sus características debería, de algún modo, formar parte de las consecuencias de ese contexto. Así, el hecho sorprendente debería hacernos dudar de la adecuación de nuestros contextos $\mathrm{y}$, por tanto, requiere de un reacomodamiento de ellos, dando paso a la abducción. ${ }^{23}$ Debrock coincide en la importancia del contexto para plantear el inicio y la resolución del proceso abductivo. ${ }^{24}$

\section{Intuición y selección de hipótesis}

La abducción es la forma más débil de inferencia, que parece transgredir los principios básicos de la lógica. Es también la "amalgama perfecta" entre los aspectos lógicos y psicológicos del proceso creación científica. ${ }^{25}$ Sin embargo, los autores consultados coinciden en que es su parte lógica la que permite esbozar condiciones para aplicar el método abductivo.

En 1878, Peirce anunciaba ya la teoría de la abducción al afirmar que la hipótesis debe ser formulada como una pregunta antes de que se hagan las observaciones para comprobarla. Más adelante se ocuparía de ofrecer los mecanismos para la construcción y selección de hipótesis. Los primeros requisitos contemplados al respecto fueron que la hipótesis debe ser capaz de explicar el hecho observado, debe ser plausible y debe conducir a conclusiones verificables. Sin embargo, este razonamiento tiene un carácter también instintivo y constituye ante todo un "proceso vivo de pensamiento". La abducción significaría, en última instancia, la adopción de hipótesis como "puro poder ser". 26

Ya hemos visto que Guy Debrock habla de un "flash de entendimiento" también instinto o intuición-, el cual permite distinguir la deducción de la abducción. Para este autor ese flash no fue entendido como un problema por Peirce, sino como un ejemplo de adaptación evolutiva, constituyendo un elemento de la formación de hábitos. Hoffmann, desde un enfoque semejante, considera que el instinto lo constituyen hábitos de acción o programas de actividad que permiten reacomodarse a los contextos $y$, por tanto, se pone en movimiento cuando el hecho sorprendente provoca un desajuste respecto de un contexto determinado, dando lugar a la formación de nuevos hábitos. ${ }^{27}$ Con esta interpretación, Hoffmann procura dar la mayor racionalidad posible a la idea peirceana de instinto.

\footnotetext{
${ }^{23}$ Michael Hoffmann, ed.cit.

24 Guy Debrock, ed. cit.

25 Lúcia Santaella, ed. cit.

${ }^{26}$ Lúcia Santaella, ed. cit.

27 Guy Debrock, ed. cit.; Michael Hoffmann, ed.cit.
} 
Por su parte, Dinda L. Gorlée dice que la abducción "consiste en adivinaciones inteligentes y relevantes que implican conexiones novedosas entre pregunta $y$ solución a través de un destello de comprensión" y lo identifica con el acto de "eureka" que se vincula al hallazgo de una respuesta novedosa, de un descubrimiento. ${ }^{28}$ En el interior del investigador se producen ideas creativas que se consiguen en momentos claves de intuición abductiva y se apoyan en la analogía. Sería una especie de instinto adivinatorio, misterioso, que introduce la explicación creativa. Gorlée reconoce en esto elementos conscientes e inconscientes que conviene develar. Uwe Wirth, por su parte, desde un análisis del lenguaje, también asocia la capacidad de seleccionar y formar hipótesis con cierta idea de "adivinación" de significados, en un contexto de comprensión dado. ${ }^{29}$

Es claro, entonces, que en el proceso de abducción hay un elemento impredecible, inasible, que se asocia con la intuición, el instinto, el flash. Sin embargo, también hay factores que dan cierta lógica a este momento clave en la invención de una idea y hay condiciones que pueden volverlo controlable, lo que permite identificarlo como un método o recorrido posible para el quehacer científico.

En primer lugar, la consideración de un contexto o perspectiva precisos. Así, las hipótesis posibles tienen su límite y, por tanto, encuentran su lógica en la interacción de los diversos contextos que están presentes en una situación históricamente determinada. ${ }^{30}$ Ninguna proposición puede ser considerada -ni en su faz lógica ni en su faz semiótica- aislada de su contexto y, en términos pragmáticos, ningún hábito puede desvincularse de la red de acciones de la que forma parte. Formular una hipótesis, una conjetura, es lanzar a un tiempo una variedad de elementos que están en gran medida determinados por un proyecto en curso, responden a una perspectiva y suponen una reorganización de los hechos. ${ }^{31}$ Esto reduce notablemente los márgenes de arbitrariedad y de azar.

Lo expuesto precedentemente nos enfrenta a la cuestión de la selección de hipótesis. Al respecto, consideramos un punto de partida interesante el que propone Hoffmann. Este autor sugiere no plantearse el problema de formular una hipótesis como creada de la nada, sino considerar que existe un número infinito de hipótesis posibles, dentro de las cuales debemos escoger la hipótesis explicativa más apropiada. ${ }^{32}$ De esta forma, proporciona un enfoque que nos parece operativo $^{33}$ para desarrollar un método abductivo que permita al científico

\footnotetext{
28 Dinda L. Gorlée, “¡Eureka! La traducción como un descubrimiento pragmático”, en Anuario Filosófico XXIX/3, ed. cit.

${ }^{29}$ Uwe Wirth, "El razonamiento abductivo en la interpretación según Peirce y Davidson", en CS Peirce y la abducción..., ed. cit.

30 Michael Hoffmann, ed. cit.

${ }^{31}$ Guy Debrock, ed. cit.

32 Michael Hoffmann, ed. cit.

33 A nuestro juicio, la formulación de una única hipótesis resulta una empresa excesivamente dificultosa que, en la práctica, puede conducir a la parálisis o al abandono del trabajo creativo; por el contrario, partir de suponer infinitas opciones, para ir descartando luego las menos adecuadas, resulta más accesible, siempre que se aplique el método adecuado para dar confiabilidad al proceso.
} 
controlar el proceso de creación hipótesis. Creemos que, en este sentido, Hoffmann está próximo a la posición de Guy Debrock, que recupera el pensamiento de Nicolás de Cusa para hacer una relectura de Peirce. De Cusa afirmaba que, al ser la verdad infinita, derivada de la infinitud de Dios, ninguna proposición humana puede ser más que una conjetura, una adivinación. Cada conjetura es en parte verdadera y en parte falsa, a la vez que hay innumerables conjeturas igualmente verdaderas y falsas. Nuestra función sería, a través de la experiencia, eliminar progresivamente aquéllas que son erróneas y acercarnos a la que más se aproxima a la verdad o a esa fracción de verdad que somos capaces de comprender. ${ }^{34}$ Estaríamos entonces ante el problema, no tanto de construir la hipótesis más adecuada, sino de elegirla dentro del abanico de posibilidades que se abre a la inteligencia cuando se haya en la búsqueda propia de la labor científica. Por otro lado, en la selección debemos considerar cuáles son las condiciones que hacen que una hipótesis sea más pertinente que otra; y qué camino, forma o método puede ser el más correcto para llegar al momento abductivo.

Existen, por un lado, condiciones ajenas a la hipótesis pero que coadyuvan a su elección. En primer lugar, la inferencia abductiva adquiere sentido en el trabajo de una mente entrenada, es decir, capacitada para llevar adelante la investigación que se propone considerando la coincidencia de factores internos al científico incluso genéticos- y externos, en el momento oportuno. La construcción y / o descubrimiento del conocimiento se produce en el interior del investigador y da cuenta de una parte singular de experiencia. ${ }^{35}$ Requiere, por tanto, una mente disciplinada y fértil, que haya desarrollado su habilidad profesional en un campo determinado. Más que la genialidad de un individuo extraordinario, Peirce enfatiza la idea de un hábito de trabajo, una disciplina de estudio, un bagaje previo de conocimientos que proporcionen las bases para realizar un hallazgo.

Asimismo, las nuevas ideas pueden ser respuestas casuales a una pregunta, que se dan en el interior, en la meditación de una mente entrenada, pero que deben ser plausibles, relevantes en relación al contexto y coherentes. Precisamente, algo es plausible, en términos pragmáticos, cuando es contextualmente relevante y refleja una coherencia lógica, a la vez que puede organizarse de manera económica y sencilla. En palabras de Peirce, esto significa seleccionar aquella hipótesis que suponga "economía de dinero, tiempo, pensamiento y energía", lo cual facilita el procedimiento para su comprobación. ${ }^{36}$

Por tanto, el criterio para que una hipótesis resulte válida supone su belleza y utilidad, es decir, su capacidad de presentar las cualidades de sencillez, unicidad,

\footnotetext{
${ }^{34}$ Según Debrock, hay notables coincidencias entre Nicolás de Cusa y Ch. Peirce que justifican una lectura de Peirce desde una perspectiva cusanesca: para ambos, existe una verdad independiente del conjunto de conjeturas; aunque esa verdad no depende de la conjetura, requiere necesariamente de ella para su revelación; la experiencia sería la herramienta para ir despejando conjeturas erróneas; y ambos se interesan particularmente por la íntima relación que ven entre epistemología y semiótica. (Cfr. Guy Debrock, ed. cit.)

35 Dinda L. Gorlée, ed. cit.

36 Uwe Wirth, ed. cit.
} 
drama, iniciativa y, a la vez, su posibilidad de ser comprobada experimentalmente; a ello hay que sumarle un tercer elemento, su fuerza explicativa fundada en la intuición. ${ }^{37}$

\section{Los pasos a seguir}

El momento de la intuición abductiva puede ser el resultado de una reorganización de los hechos. Hemos visto que para Peirce todo pensamiento se da mediante signos y una proposición es un símbolo. Como todo signo, los símbolos deben tener un objeto y un interpretante. $Y$ todo enunciado categórico es una hipótesis o conjetura. Según Debrock, el objeto de tal símbolo proposicional es una conjetura tal como la planteó Nicolás de Cusa: lanzar conjuntamente diversos elementos. Y su interpretante, a la vez, está determinado por el proyecto en curso, el cual, por su parte, determina cómo el símbolo proposicional representa la reorganización de los hechos. La idea que aporta este autor -que toma de de Cusaes que todo símbolo está relacionado con una conjetura. Por tanto, reorganizar los hechos supone cambiar radicalmente la perspectiva desde la cual esos hechos han sido organizados, incluso en el sentido de cambio gestáltico; e implica realizar un acto de simbolización que proporciona una perspectiva absolutamente nueva que, a su vez, remite a una conjetura que es la base de toda inferencia abductiva. La conjetura sería, de este modo, un symbolon capaz de unificar los hechos desde una perspectiva novedosa. ${ }^{38}$

Algunos autores sugieren pasos y recursos para llevar a cabo este reordenamiento del modo más eficaz posible. Hemos dicho que se debe partir de contar con ciertas condiciones en el investigador, ante todo, su entrenamiento, idoneidad y disciplina. Gorlée recomienda la suspensión temporal de toda actividad voluntaria e ignorar las rutinas lógicas para enfrentarse desinteresadamente al problema, sin prejuicios mentales. Hay que observarlo desde diversas perspectivas, en sus múltiples posibilidades, en toda su dimensión. ${ }^{39}$ El investigador debería poder jugar y experimentar. Debrock propone jugar con el material disponible, experimentar con él, como hacen el artista o el artesano con su obra, hasta encontrar un concepto, un símbolo, que unifique los elementos, que les dé nueva forma. Para este autor, el impulso del juego tendría una función mediadora que representa la terceridad. Gorlée recomienda la experimentación mediante diagramas, buscando combinar los elementos de forma significativa y eficaz para resolver el problema. Las circunstancias externas también son importantes, el mínimo ruido molesto distrae o interrumpe el proceso, y requiere una acción voluntaria específica que permita recuperar el estado anterior. Finalmente, cuando la situación está madura para el

\footnotetext{
37 Dinda L. Gorlée, ed. cit.

${ }^{38}$ Guy Debrock, ed. cit.

${ }^{39}$ Dinda L. Gorlée, ed. cit.
} 


\section{María del Carmen Pía Martín}

descubrimiento, la que se espera sea la suposición más adecuada, surge de repente, como un destello de luz. Luego se produce una "epifanía abductiva", que permite liberar tensiones del cuerpo y la mente, quedando en disponibilidad para el próximo trabajo.

\section{La abducción en el método histórico}

Si tenemos presente que Peirce creía que todas las ramas de la ciencia se fundan en un razonamiento lógico y que para el progreso científico es necesario el desarrollo de una teoría general de la lógica ${ }^{40}$, se entiende la importancia de profundizar el estudio de la abducción aplicada al campo de las Ciencias Sociales. Hemos visto que el objeto de las Ciencias Sociales, y en particular de la historia, ha de considerarse uno de los tipos de hechos sobre los que nuestro autor propone hipotetizar.

Todas las Ciencias Sociales, en tanto se refieren al hombre y a la sociedad, se ven afectadas por la variable temporal. En el caso específico de la historia, ella se remite sobre todo a aquellos acontecimientos, procesos, acciones, personas, instituciones y estructuras que se han extinguido en el tiempo y que no pueden recrearse más que en la mente del historiador. Y es gracias a los restos testimonios y documentos- que quedan del pasado, que el investigador tiene indicios de una realidad cuya existencia, siempre problemática, es posible y verificable. Es decir, estamos frente a una realidad o, mejor, un recorte de la realidad que no puede ser observado directamente, pero que puede ser reconstruido e interpretado a través de sus vestigios materiales e, incluso, reactualizado en el presente mediante el trabajo del especialista, como quería Croce. Asimismo, la practicidad de la historia está dada sobre todo por la necesidad que tiene todo grupo humano de tener conciencia de su pasado colectivo $\mathrm{y}$, en este sentido, forma parte constitutiva del presente, no para predecirlo sino para comprenderlo.

A nuestro juicio, el historiador siempre interroga al pasado desde sus preocupaciones del presente, lo que implica que el conocimiento del pasado nunca puede darse por clausurado y que los mismos hechos, procesos o fenómenos, pueden ser enfocados desde otras perspectivas, planteando problemas inéditos $y$, por tanto, sugiriendo nuevas hipótesis. Por otro lado, en las Ciencias Sociales hay una mayor implicación de la subjetividad del científico -particularmente acentuada en el caso de la historia- quien, para explicar las operaciones realizadas por sujetos de los que está separado temporal y espacialmente, debe realizar una reproducción o reactualización analógica de esas mismas operaciones, ${ }^{41}$ para luego tomar distancia y marcar especificidades y diferencias, evitando los anacronismos.

\footnotetext{
${ }^{40}$ Michael Hoffmann, ed. cit.

41 Enrique Moradiellos, El oficio de historiador, Barcelona, Siglo XXI, p. 6.
} 


\section{Abducción, método científico e Historia}

Debido a que las cuestiones del presente nos llevan a interrogarnos sobre el pasado desde otras perspectivas, a la vez que ese pasado en muchos aspectos sigue siendo un componente del presente que nos preocupa; y debido también a la singularidad de los hechos pretéritos, que no pueden repetirse, la utilización de la hipótesis es clave para el método histórico. La historia se presenta actualmente como una ciencia interpretativa y, en este sentido, nos parece de particular interés recurrir al aporte metodológico de Charles Peirce. En cuanto al concepto de abducción, consideramos tan relevante para nuestra disciplina destacar el carácter explicativo de la hipótesis, como considerar la recurrencia a mecanismos analógicos implícitos en ciertas abducciones, si se quiere imperfectas o débiles -en términos de Debrock-, pero necesarias para una disciplina que pretende comprender a las sociedades humanas extintas desde su propia humanidad.

Evidentemente, toda ciencia que procure ser explicativa y avanzar con nuevos enfoques sobre cuestiones muchas veces estudiadas en extenso, deberá apropiarse del método abductivo en sentido peirceano. Cuando el historiador suficientemente entrenado, con una formación teórica adecuada, descubre una incongruencia, un vacío aparentemente inexplicable, una contradicción en su objeto de estudio, en el marco de un contexto determinado, está ante un hecho sorprendente que, a su vez, cuestiona sus propias creencias o convicciones al respecto. Se enfrenta a un problema que lo mueve a actuar para encontrar la explicación más adecuada. Ese hecho sorprendente puede ser un desajuste del presente que lo remite al pasado o puede hallarlo en el pasado, aunque con proyección al presente. Muchas veces buscamos una mejor explicación de un fenómeno o proceso, tratando de indagar el por qué, considerando la más amplia diversidad de causas posibles, complejizando el entramado de relaciones entre ellas. Otras veces accedemos al problema recurriendo a razonamientos analógicos cuyos aspectos inapropiados serán progresivamente descartados, a medida que avancemos en la fase de verificación. El historiador puede partir de conjeturas casuales, fundadas sin embargo en su formación y conocimiento previos, y en los desajustes que es capaz de detectar; o partir de cierta abstracción analógica que le permita acercarse a un tiempo que se ha extinguido, para luego diferenciar y acotar la cuestión.

Nos parece adecuado, como propone Hoffmann, no tanto buscar una única hipótesis, sino considerar un sinnúmero de hipótesis posibles, sopesando todos los elementos disponibles, combinándolos, jugando con ellos, para finalmente descartar aquellos elementos y relaciones que parezcan más inconsistentes improbables ante la compulsa de la documentación disponible- y reforzando las relaciones y formas verificables, hasta dar con el concepto que los unifique en una nueva perspectiva más coherente. A veces, además del ambiente externo y el silencio interior, un momentáneo distanciamiento del problema que nos ocupa crea las condiciones adecuadas para, al retomar el trabajo, encontrar la respuesta que buscábamos. Ése es el momento en que la hipótesis se presenta como en un flash de entendimiento, intuitivamente da la sensación de ser la más adecuada por 
su sencillez, economía, verificabilidad, plausibilidad. Deben entonces considerarse sus derivaciones posibles y comenzar el proceso de comprobación empírica que, en nuestro caso, significa volver a las fuentes, recuperar una información muchas veces fragmentaria -el historiador suele tener vacíos irrecuperables por la misma naturaleza de su objeto-, pero ahora ordenada y jerarquizada según su relevancia para afirmar, corregir o descartar la hipótesis propuesta.

Por otro lado, aunque esto excede el propósito del presente trabajo, creemos que el método abductivo puede también emplearse para el abordaje de documentos escritos y, en el caso de estudios más contemporáneos, de testimonios. Lógicamente, estas fuentes suelen corresponder a sociedades distintas a la propia, a épocas más o menos alejadas de la nuestra y suponen experiencias, prácticas, representaciones, usos e intencionalidades que nos son desconocidos. Podría aplicarse aquí el análisis que propone Uwe Wirth, de tal modo que la interpretación de fuentes no debería suponer una mera aplicación de códigos y convenciones, sino que podría considerarse un proceso inferencial de adopción de hipótesis sobre la intención y los significados posibles. A la vez que permitiría descubrir reglas prácticas para crear nuevas teorías. La abducción sería entonces un óptimo recurso para la interpretación de fuentes, tomada como un proceso de reconstrucción inferencial de causas e intenciones, tanto como la construcción inventiva de teorías aplicada a la interpretación del texto histórico. ${ }^{42}$

\section{Conclusión}

En el presente trabajo hemos procurado hacer una confrontación de textos que nos permitieran comprender el concepto de abducción formulado por Charles Peirce desde finales del siglo XIX. Hemos seguido, en la medida de lo posible, el recorrido intelectual que le permitió definir la abducción como una forma de inferencia distinta y, sobre todo, como el primer paso del método científico. Esto ha permitido resaltar la centralidad de la formulación de hipótesis para el progreso de la ciencia y afirmar la existencia de una lógica abductiva, la cual posibilita un método que haga controlable la búsqueda de ideas nuevas, necesarias para la invención y el descubrimiento científicos. El carácter más débil de la abducción como forma de inferencia se compensa sobradamente con su naturaleza explicativa y ampliativa, capaz de gestar nuevas ideas y teorías, a tal punto que constituye el modo de razonamiento creativo por excelencia.

Hemos considerado que, en el estado actual de la ciencia histórica, cuando se ha superado hace ya mucho la vocación descriptiva, y se pretenden nuevas interpretaciones y explicaciones más exhaustivas, recurriendo a enfoques, fuentes, perspectivas antes impensados, tanto la aplicabilidad como la practicidad del método abductivo resultan evidentes. Hemos planteado su utilidad en dos

${ }^{42}$ Cfr. Uwe Wirth, ed. cit. 
sentidos. Por un lado, por su aporte metodológico para la búsqueda de nuevas hipótesis. Esto puede darse como una conjetura casual o partiendo de abstracciones de tipo analógicas, en ocasiones la forma más común. La búsqueda de una explicación a través del planteo de la hipótesis adecuada puede surgir dentro de un abanico de posibilidades, descartando aquéllas que no reúnan las condiciones de economía y plausibilidad. Poder jugar, experimentar, especular con los elementos disponibles hasta dar con la respuesta adecuada es parte del proceso de todo trabajo científico. Por último, la deducción de consecuencias y su comprobación empírica completan el proceso, aunque éste recién se cierra cuando se somete al juicio de la comunidad académica, o quizás se abre, porque siempre queda sujeto a sus sugerencias $y$, por tanto, a nuevas revisiones.

Por otro lado, propusimos aplicar el método abductivo a la interpretación de fuentes, aspecto clave para una correcta reconstrucción de nuestro objeto de estudio, buscando aprehender el sentido y las intencionalidades más apropiadas, sin incurrir en anacronismos ni en ucronías, pero desarrollar un análisis de esta magnitud excede las posibilidades del presente trabajo.

\section{Bibliografía}

Anuario Filosófico XXIX/3, 1996, http://www.unav.es/gep/AF/AFIndice.html

Peter Burke, Formas de hacer historia, Madrid, Alianza, 1993.

C. S. Peirce y la abducción. Analogía Filosófica XII/1, 1998, 1-187 http://www.unav.es/gep/AN/ANIndice.html

Guillermo Brion, Método y Técnicas, México, Editorial Trillas, 1995.

Ciro Cardozo y Héctor Pérez Brignoli, Los métodos de la Historia, Barcelona, Crítica, 1984.

Edward H. Carr, ¿Qué es la Historia?, Barcelona, Planeta-Agostini, 1993.

Esther Díaz (editora), La posciencia. El conocimiento científico en las postrimerías de la modernidad, Buenos Aires, Editorial Biblos, 2004.

Eric Hobsbawm, Sobre la Historia, Barcelona, Crítica, 1998.

Enrique Moradiellos, El oficio de historiador, Madrid, Siglo XXI, 1994.

Charles S. Peirce, Obra lógico-semiótica (edición de Armando Sercovich. Versión castellana de Ramón Alcalde y Mauricio Prelooker), Madrid, Taurus, 1987.

Charles S. Peirce, Sobre la lógica de la extracción de la Historia a partir de Documentos Antiguos, especialmente testimonios, 1901. Traducción castellana de Douglas Niño,

2001.

https://www.google.com.ar/?gws rd=ssl\#q=Sobre+la+L\%C3\%B3gica+de+la+Extr acci\%C3\%B3n+de+la+Historia+a+partir+de 


\section{María del Carmen Pía Martín}

Charles Peirce, Lecciones de Harvard sobre el pragmatismo. Lección VI: Tres tipos de razonamiento, 1903. Traducción al castellano y notas de José Vericat, 1988, http://www.unav.es/gep/OnThreeTypesReasoning.html

Juan Samaja, “Aspectos lógico-epistemológicos”, en Pensar y hacer la investigación, Editorial Docencia, Buenos Aires, 2002.

Félix Schuster, El método en las Ciencias Sociales, Buenos Aires, CEAL, 1992.

Recibido: 18/06/2015

Evaluado: $24 / 07 / 2015$

Versión Final: 20/08/2015 